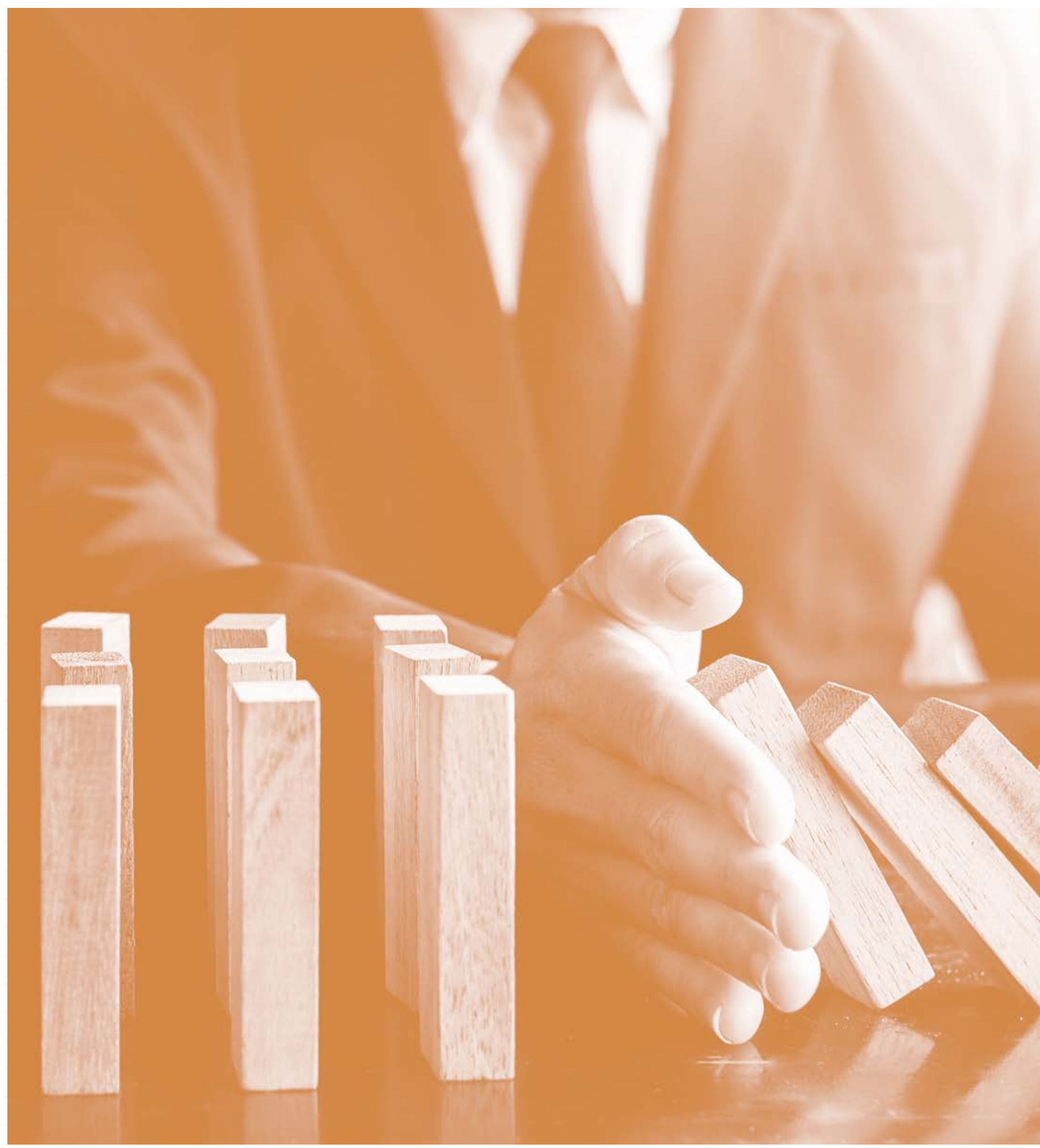

\title{
.98
}




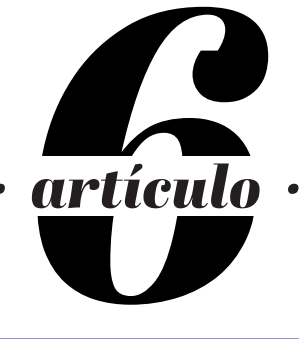

\section{Modelos de incorporación de los derechos económicos, sociales, culturales y ambientales: Aprendizajes para el proceso constituyente chileno.}

Dra. Katie Boyle ${ }^{184}$, Vicente Silva ${ }^{185}$ y Valentina Contreras Orrego 186 DOI: 10.53110/PWVG6458

A. Introducción: La incorporación del derecho internacional de los derechos humanos en el derecho nacional significa incorporar los estándares legales dispuestos en el derecho internacional e implementarlos a nivel doméstico. Si bien este proceso de incorporación puede tomar muchas formas, este documento intenta presentar algunas maneras mediante las cuales Chile podría incorporar los derechos económicos, sociales, culturales y ambientales (DESCA) en el marco normativo nacional, aprovechando el momento constituyente

\footnotetext{
183 Artículo originalmente preparado por Katie Boyle, para la Comisión de Derechos Humanos de Escocia.

184 Abogada de derecho constitucional y derechos humanos. Profesora asociada de Derecho Internacional de los Derechos Humanos de la Universidad de Stirling, Reino Unido.

185 Abogado, Universidad de los Andes. Magíster en derecho internacional de los derechos humanos de la Universidad de Essex, Reino Unido. Actualmente se desempeña como representante en Latino América de la Iniciativa Global por los Derechos Económicos, Sociales y Culturales. 186 Abogada de la Universidad de Chile, MSc en Derechos Humanos por la London School of Economics and Political Science (LSE) y representante en Chile de la Iniciativa Global por los Derechos Económicos, Sociales y Culturales (GI-ESCR).
} 
en que se encuentra. Tradicionalmente, la incorporación se ha entendido como una manera directa de recoger el derecho internacional en el sistema legal nacional a través de la Constitución o mediante legislación nacional. Sin embargo, este documento adopta una visión más amplia y completa de la incorporación, incluyendo una variedad de métodos mediante los cuales se introducen tanto los estándares legales internacionales como los remedios eficaces al marco normativo nacional. Por lo tanto, este documento se centra en los modelos de incorporación por un lado y en los mecanismos de justiciabilidad por el otro. ${ }^{187}$

La incorporación y justiciabilidad de los DESCA no es un fenómeno nuevo, ha ocurrido por todo el mundo de maneras diferentes. Este documento presenta caminos potenciales de cambio constitucional, para que los procesos de reforma con enfoque de derechos humanos sigan elaborándose a partir de datos, evidencia comparada y las mejores prácticas.

Al respecto, algunos países ya han realizado avances sustantivos para incorporar el derecho internacional de los derechos humanos en su legislación nacional, tales como Noruega, Bélgica y España. Más recientemente, se destaca Suecia188 -con su propuesta de incorporar 'los principios de la Convención sobre los Derechos del Niño de las Naciones Unidas'-189 y Escocia, que instituyó el Grupo Consultivo del Primer Ministro para considerar cómo este país podría seguir siendo un líder en materia de derechos económicos, sociales, culturales y ambientales, a la vez que para revisar la incorporación de estos derechos en la legislación doméstica. ${ }^{190}$ Este Grupo Consultivo recomendó incorporar los DESC para facilitar las discusiones sobre la manera en que Escocia traduce la legislación internacional de los derechos humanos en el ámbito nacional, lo que derivó en la creación de un órgano especial para aplicar estas recomendaciones y preparar una nueva Ley sobre los Derechos Humanos en el país. Esta experiencia, así como otras, entregan diversas lecciones para otros procesos constitucionales, entre las que se pueden extraer valiosos aprendizajes para el proceso constituyente en Chile.

\footnotetext{
187 Véase, por ejemplo, la definición de incorporación desarrollada por el Comité de Derechos del Niño, 2003.

188 La nueva legislación aprobada en Suecia el $1^{\circ}$ de enero de 2020 incorporó la CDN en el ámbito nacional. Disponible en: https://www.government.se/articles/2018/04/questions-and-answers-about-incorporating-un-conventionon-the-rights-of-the-child-into-swedish-law/ (Consulta: 14 diciembre 2020).

189 Scottish Government, sin fecha.

190 First Minister's Advisory Group on Human Rights Leadership, sin fecha.
} 
Los derechos humanos incluyen a los derechos civiles, políticos, económicos, sociales, culturales y ambientales. En términos amplios, la expresión derechos civiles y políticos se ha entendido tradicionalmente como la garantía de las libertades individuales frente a la intervención gubernamental, 191 mientras que la expresión derechos sociales se utiliza para denominar a la categoría de derechos de igualdad que se refieren al bienestar económico y social, ${ }^{192}$ constituyendo un posible medio para promover un mayor grado de justicia social. ${ }^{193}$ En este contexto, los derechos civiles y políticos incluyen derechos como el derecho a un juicio imparcial, el derecho a votar o la libertad de tránsito, entre otros; mientras que los DESCA incluyen derechos como la educación, la seguridad social, condiciones justas de trabajo, la vivienda adecuada, el derecho a la salud y el derecho a la alimentación, entre muchos otros.

El tratamiento separado de los derechos civiles y políticos y los DESCA obedeció a un método de trabajo fijado por la naciente Organización de Naciones Unidas, altamente influenciada por las ideologías de la guerra fría vigentes en la época. ${ }^{194}$ Sin embargo, con el tiempo se fue instalando la discusión en torno a la unidad de los cimientos de ambos derechos alrededor de los valores de la dignidad, libertad y pertenencia a la comunidad, ${ }^{195}$ situación que fue zanjada en 1993 a través de la Declaración y Programa de Acción de Viena, la que codificó el principio de indivisibilidad de los derechos, destacando que:

"Todos los derechos humanos son universales, indivisibles e interdependientes y están relacionados entre sí. La comunidad internacional debe tratar los derechos humanos en forma global y de manera justa y equitativa, en pie de igualdad y dándoles a todos el mismo peso". 196

Sin embargo, históricamente el estatus legal de los DESCA ha continuado siendo malinterpretado, ${ }^{197}$ derivando en una falta de entendimiento sobre la forma en que los DESCA deben ser aplicados en la práctica. ${ }^{198}$

191 De Búrca, 2012, p. 3.

192 Ibíd., p. 4.

193 Ibíd., p. 4.

194 Gearty y Mantouvalou, 2011, p. 98.

195 Ibíd., p. 98.

196 Conferencia Mundial de Derechos Humanos, 1993, p. 19.

197 Craven, 1995.

199 Sobre las discusiones relativas a los conceptos erróneos que rodean la dicotomía de los derechos humanos basados en una antinomia positiva vs. negativa, véase: Koch, 2005.
B.

El estatus legal
de los DESCA y la
"justiciabilidad"
de los derechos

El estatus legal de los DESCA y la de los derechos 
Las sucesivas medidas para proteger los derechos humanos, tanto a nivel regional como nacional, se han centrado erróneamente sólo en los derechos civiles y políticos, relegando los DESCA a meras aspiraciones, objetivos políticos o asuntos cuya regulación depende exclusivamente del poder legislativo. ${ }^{199}$ Esta idea equivocada surge por la comprensión generalizada de que los derechos civiles y políticos son exigibles ante los tribunales de justicia -es decir, son "justiciables"-, en contraposición a los DESCA, en cuya aplicación "con demasiada frecuencia se parte del supuesto contrario". 200 Así, cuando un Estado ha incorporado los derechos civiles y políticos en su marco constitucional, los tribunales pueden intervenir para proporcionar un remedio cuando éstos han sido violados, situación que no siempre sucede con los derechos sociales. Lo anterior, ha creado una brecha en términos de la rendición de cuentas del Estado en relación con la garantía de los derechos no incluidos expresamente en el texto constitucional. Sin embargo, han pasado más de tres décadas desde que la literatura y las mejores prácticas internacionales han reconocido que los DESCA son normas legales internacionales vinculantes y susceptibles de ser ejecutadas judicialmente. ${ }^{201}$

De esta manera, el Comité de Derechos Económicos, Sociales y Culturales (el órgano de Naciones Unidas responsable de supervisar la aplicación del Pacto Internacional de Derechos Económicos, Sociales y Culturales) ha requerido el aseguramiento de remedios judiciales ${ }^{202}$ ante violaciones de los DESCA. ${ }^{203}$ Conforme al criterio del Comité, la diferenciación en la protección judicial de los derechos civiles y políticos y los DESCA "no está justificada ni por la naturaleza de los derechos ni por las disposiciones pertinentes del Pacto", ${ }^{204}$ agregando que la negativa generalizada a reconocer la naturaleza justiciable de los DESCA es arbitraria debiendo entonces recibir la misma protección que los derechos civiles y políticos en el marco normativo nacional. ${ }^{205}$ Las discusiones doctrinarias y jurisprudenciales más relevantes hoy giran en torno a cuál es la mejor forma de proporcionar remedios ejecutables o a través de qué mecanismos podrían incorporarse mejor los DESCA dentro de un marco constitucional particular, de forma factible y legítima.

\footnotetext{
199 Op. Cit., Craven, 1995.

200 Comité de Derechos Económicos, Sociales y Culturales, 1998, p. 4.

201 Véase: Gauri y Brinks, 2010 y Minkler, 2013.

202 Para efectos de este documento, el término "justiciabilidad" se referirá a la adjudicación de un derecho por parte de un tribunal imparcial.

203 Op. Cit., Comité de Derechos Económicos, Sociales y Culturales, 1998, pár. 10.

204 Ibíd., pár. 10.

205 Ibíd., pár. 10.
} 
La incorporación de los DESCA implica adoptar a nivel doméstico los estándares internacionales que obligan a los Estados. Esto puede ocurrir a través de una variedad de vías o "puertos"206 tales como: (i) la constitucionalización de las normas internacionales, (ii) la adopción legislativa o administrativa de normas internacionales de derechos humanos, (iii) la suscripción de un mecanismo internacional de quejas y el cumplimiento de sus decisiones a nivel nacional, o (iv) la incorporación de un enfoque judicial del derecho consuetudinario. ${ }^{207}$ Estas vías forman parte de los diversos componentes básicos utilizados en diferentes modelos constitucionales y no son mutuamente excluyentes: el derecho internacional de los derechos humanos puede integrarse a través de estas diferentes vías de forma aislada o simultáneamente. En cualquier caso, la incorporación requiere de la vernacularización de estándares legales establecidos en el derecho internacional, para hacerlos aplicables al contexto nacional específico.

A pesar de que la incorporación de los DESCA no es una bala de plata para resolver todos los problemas sociales, su reconocimiento es una piedra angular para guiar el desarrollo progresivo de legislación y políticas públicas que permitan un mejor acceso a los derechos directamente relacionados con las condiciones de vida de las personas. ${ }^{208}$ Además, permite establecer un marco de rendición de cuentas que obligue a los poderes públicos a considerar el derecho internacional de derechos humanos en el desempeño de sus funciones.

En Chile, la Constitución vigente reconoce parcialmente algunos DESCA, entre los que se incluyen el derecho a vivir en un medio ambiente libre de contaminación ( $\left.N^{\circ} 8\right)$, el derecho a la protección de la salud $\left(N^{\circ} 9\right)$, el derecho a la educación ( $\left.\mathrm{N}^{\circ} 10\right)$, los derechos de los trabajadores $\left(\mathrm{N}^{\circ} 16\right)$, el derecho a la seguridad social ( $\left.\mathrm{N}^{\circ} 18\right)$ y el derecho a sindicarse $\left(\mathrm{N}^{\circ} 19\right)$. Sin embargo, a nivel constitucional el Estado no asume un rol activo en la provisión de servicios públicos, sino que le entrega a los actores privados la responsabilidad de satisfacer los DESCA a través de estrategias de mercado. ${ }^{209}$ Lo anterior queda de manifiesto al revisar el artículo 20 de la Constitución, el que contempla un recurso judicial de protección en caso de violación de algunos de los derechos contemplados en el artículo 19, cautelando así la intervención de los privados en la provisión pública. Es así que el acceso a este recurso está disponible sólo en ciertas circunstancias, por ejemplo, la protección del derecho a la salud sólo respecto de las violaciones del derecho a elegir
C.

¿Qué queremos

decir con incorporación?

206 Resnik, 2006, pp. 1564-1670.

207 Ibíd.

208 Véase: Scottish Human Rights Commission, 2015.

209 Figueroa y Jordán, 2020. 


\section{9}

Abordando estos temas, el Comité de Derechos Económicos, Sociales y Culturales de la ONU ha señalado que la actual consagración constitucional no cumple con los estándares internacionales de derechos humanos, mostrando su preocupación porque "que el recurso de protección establecido en la Constitución deja fuera de la protección jurídica algunos de los derechos contenidos en el Pacto, limitando su aplicabilidad directa por los tribunales nacionales". 211 el sistema de atención sanitaria (público o privado), lo que desplaza el derecho al disfrute del más alto nivel posible de salud en los términos establecidos en el derecho internacional. ${ }^{210} \mathrm{Si}$ milarmente, si bien el artículo $19 \mathrm{~N}^{\circ} 10$ incorpora el derecho a la educación, la referida acción judicial sólo puede ser interpuesta respecto de las violaciones a la libertad de los padres de elegir el establecimiento educacional de sus hijos $\left(19 \mathrm{~N}^{\circ}\right.$ 11), transformando el derecho a la educación en un derecho de segunda categoría.

Abordando estos temas, el Comité de Derechos Económicos, Sociales y Culturales de la ONU ha señalado que la actual consagración constitucional no cumple con los estándares internacionales de derechos humanos, mostrando su preocupación porque "que el recurso de protección establecido en la Constitución deja fuera de la protección jurídica algunos de los derechos

contenidos en el Pacto, limitando su aplicabilidad directa por los tribunales nacionales". 211 Por lo anterior, recomendó al Estado de Chile garantizar "el reconocimiento integral y la protección jurídica necesaria de los derechos económicos, sociales y culturales en el nuevo texto constitucional, asegurando que el proceso de reforma constitucional previsto se realice de una forma transparente y participativa". ${ }^{212}$

En este sentido, la incorporación nacional de las normas debiera basarse en el marco normativo internacional e ir acompañada en todo momento de un remedio efectivo ante la violación de un derecho. Si bien los remedios pueden adoptar formas muy diversas, cualquier debate sobre cambios constitucionales relativos a los DESCA debería tener en cuenta estrategias que privilegien remedios innovadores. Así, la oportunidad de elaborar una nueva Constitución en Chile permite clarificar y expandir las vías para proporcionar remedios efectivos en el contexto de los DESCA a través, por ejemplo, de las acciones de protección y la litigación colectiva.

\footnotetext{
210 Pacto Internacional de Derechos Económicos, Sociales y Culturales, artículo 12

211 Comité de Derechos Económicos, Sociales y Culturales, 2015, pár. 7.

212 Ibíd., pár.7.
} 
Aprovechando el proceso constituyente en marcha, Chile tiene la posibilidad de extraer lecciones de otras experiencias, pudiendo convertirse en un líder internacional si logra desarrollar formas más innovadoras de incorporar los DESCA en su Constitución, por ejemplo, con la adopción de medidas de igualdad sustantiva, como ya lo ha hecho a través de la elección de la primera convención paritaria del mundo.

Las mejores prácticas comparadas evidencian que la incorporación doméstica debe producirse a través de las instituciones políticas, lo que significa que las ramas legislativa, ejecutiva y judicial deberían estar vinculadas por los mismos principios y actuar como garantes de los derechos humanos, recogiendo así el planteamiento multiinstitucional de los DESCA que reconoce la importancia de involucrar a todas las ramas del Estado en las decisiones que afectan estos derechos. Por ello, los modelos que a continuación se analizan pretenden identificar los roles que las diferentes instituciones gubernamentales juegan en la incorporación doméstica de los estándares internacionales.

Los modelos constitucionales pueden variar significativamente de un país a otro, lo que influye en la diversidad de mecanismos que garantizan el acceso a los derechos. Esto significa que existe una amplia gama de estrategias adoptadas por los Estados para proteger las garantías constitucionales. Así, los mecanismos de incorporación doméstica pueden ofrecer diferentes grados de aplicación de la ley, variando desde mecanismos de supremacía parlamentaria, tribunales muy reticentes a interferir en la garantía de derechos (se muestran deferentes con el parlamento/gobierno) hasta tribunales que actúan de manera significativa para proteger los derechos (defienden la Constitución y actúan como garantes de los derechos humanos). Estas estrategias son muy importantes, en tanto entregan información sobre los distintos tipos de incorporación normativa que pueden existir.

\section{Finlandia}

En Finlandia, los DESCA reciben protección constitucional. Sin embargo, todas estas disposiciones están dirigidas al Poder Legislativo: el derecho a la ciudadanía (artículo 5); el derecho a la igualdad ante la ley (artículo 6); los derechos educativos (artículo 16); el derecho a la lengua y la cultura (artículo 17); el derecho al trabajo (artículo 18) y el derecho a la seguridad social (artículo 19), requieren que se les dé efecto a través de una legislación subsecuente. ${ }^{213}$

213 Las disposiciones constitucionales estipulan que los detalles acerca de cómo se garantizarán los derechos deben ser proporcionados por ley.
D.

Modelos
constitucionales
comparados


En este sentido, la Constitución impone una obligación preceptiva al Poder Legislativo para que regule la protección y el cumplimiento de los DESCA. En este contexto, el modelo finlandés se encuentra acorde a la doctrina de la supremacía parlamentaria, dejando a salvo la posibilidad de que exista una aplicación judicial directa o control de convencionalidad por parte de los tribunales de justicia. Sin embargo, el Comité DESC ha lamentado la falta de información sobre casos de aplicabilidad directa de la convención frente a los tribunales domésticos, ${ }^{214}$ lo que podría hacer suponer que el control judicial ex post aún no está suficientemente desarrollado.

\section{Colombia}

La Constitución Colombiana divide a los derechos humanos en tres grupos: derechos fundamentales, sociales y derechos colectivos y medioambientales (capítulos 1, 2 y 3). Dentro de los derechos sociales incluidos en la Constitución se encuentran la salud, la vivienda, el trabajo y la educación, entre otros. A su vez, se protege a los grupos vulnerables y desfavorecidos de la sociedad con medidas particulares para los niños, las mujeres, las personas mayores y las personas con discapacidad (artículos 46-47). Esta Constitución incorpora directamente en la legislación doméstica los derechos garantizados en los tratados internacionales ratificados por Colombia (artículo 44), equiparando así a los tratados de derechos humanos con una norma constitucional a través de su prevalencia en el orden interno (artículos 93).

Para hacer operativa esta prevalencia, la Carta Colombiana consagra la acción de tutela (artículo 86) como el principal mecanismo para su protección jurídica. La tutela permite que una persona presente una solicitud de protección ante cualquier juez de la República para que se decrete la protección inmediata de sus derechos constitucionales fundamentales. Sin perjuicio de la aplicabilidad inmediata del fallo del juez correspondiente, todas las decisiones emitidas por jueces ordinarios en sede de tutela se envían a la Corte Constitucional para su eventual revisión. Los magistrados de la Corte Constitucional pueden revisar las tutelas y, cuando proceda, agrupar casos para examinar problemas estructurales. Así por ejemplo, cuando surge una problemática que afecta a un grupo grande de personas vulnerables, los casos son agrupados y la Corte determina un remedio colectivo. Esta función de la Corte Constitucional se enmarca dentro de su responsabilidad de defender la integridad y supremacía de la Constitución (artículo 241), la que se concreta en la unificación de la jurisprudencia en la materia, en aras de garantizar la igualdad y seguridad jurídica. ${ }^{215}$ Como resultado de

\footnotetext{
214 Comité de Derechos Económicos, Sociales y Culturales, 2014, pár. 6.

215 Carrera, 2011, pp. 79-80.
} 
la aplicación de la tutela, se ha producido un profundo cambio en la cultura legal del país, lográndose considerables avances en la aplicación judicial de los DESCA. ${ }^{216}$

\section{Alemania}

En Alemania, los derechos humanos asumen estatus constitucional. Los derechos constitucionales no incluyen todos los derechos económicos, sociales y culturales, sin embargo, la Constitución sí consagra un derecho a la dignidad (artículo 1.1) que la Corte ha interpretado como constitutivo de obligaciones mínimas (existenzminimum) a través de la garantía de derechos sociales particulares. ${ }^{217}$ Las responsabilidades de gobierno, incluyendo el cumplimiento de las obligaciones de los derechos humanos, ${ }^{218}$ se dividen entre un nivel federal (Bund) y un nivel descentralizado (Länder).

\section{Sudáfrica}

El modelo sudafricano adopta una mezcla de reconocimiento sustantivo de derechos humanos junto con salvaguardias y cláusulas de limitación contenidas en la Constitución. A los derechos se les concede protección en diferentes niveles con respecto a las obligaciones de respeto, protección, promoción y cumplimiento. ${ }^{219}$ Algunos derechos denominados "negativos" gozan de protección inmediata, como por ejemplo, el derecho a no ser desalojado sin un procedimiento justo. ${ }^{220}$ A algunos derechos se les otorga un estado de inderogabilidad, como por ejemplo, a los derechos relativos a los niños. ${ }^{221}$ Otros derechos son considerados como susceptibles

\footnotetext{
216 Sepúlveda, 2009, p. 144.

217 Ley Fundamental para la República Federal Alemana, modificada en 2014, artículo 1.1 .

218 Según el caso BVerfGE 125, 175 (Hartz IV).

219 Según el Artículo 7 de la Constitución.

220 Véase, por ejemplo, el caso Occupiers of 51 Olivia Road, Berea Township and 197 Main Street, Johannesburg $\vee$ City of Johannesburg and Others, CCT 24/07, [2008] ZACC 1. En dicho caso, la Corte determinó que la Constitución exige de un compromiso y participación significativos por parte de las comunidades afectadas antes que se pueda extender una orden de desalojo (no puede haber desalojos forzosos sin previo aviso).

221 Como el derecho a ser protegido contra el maltrato, el abandono, el abuso o la destitución; y el derecho a ser protegido de prácticas de explotación laboral (sección 28 (1) (d) y (e)). Para leer un análisis de los derechos del niño (en particular los derechos ESC de las niñas) en la Constitución de Sudáfrica, ver: Skelton, 2010, p. 141.
} 
de efectivización progresiva, como el derecho a acceder a una vivienda adecuada y el derecho a acceder a la atención sanitaria, a la alimentación, al agua potable y a la seguridad social. ${ }^{222}$ Adicionalmente, existe una cláusula de limitación general en el artículo 36, por la que los derechos pueden ser limitados si las referidas restricciones son razonables y justificables en una sociedad abierta y democrática. ${ }^{223}$ Más aún, la Corte Constitucional Sudafricana ha dictaminado y ha hecho valer los DESCA a través de la aplicación de un examen de "razonabilidad" a la hora de evaluar su cumplimiento por parte del Estado.

\section{Suecia}

En Suecia existe un proceso de control difuso y preventivo de la legislación por parte de los tribunales de justicia en los casos en que la ley resulte manifiestamente incompatible con la Constitución u otras provisiones de ley superior.224 Por una parte, este tipo de control ex ante de la legislación resulta positivo al dificultar (aunque no imposibilitar) la dictación de legislación que infrinja los derechos fundamentales. ${ }^{225}$ Por otro, sin embargo, hay quienes consideran que la revisión judicial es anti-democrática, en tanto parece resultar más apropiado que la decisión sobre la conformidad de una norma con la Constitución la adopte un cuerpo mayoritariamente electo como es el parlamento, ${ }^{226}$ dejando a los tribunales como un mecanismo de última instancia para garantizar que el Poder Ejecutivo y el Legislativo actúen de manera adecuada. En cualquier caso, el Comité DESC ha expresado su preocupación respecto a que el ordenamiento constitucional sueco aún no incorpora los derechos contenidos en el Pacto Internacional de Derechos Económicos, Sociales y Culturales, lo que los transforma en derechos no justiciables a nivel doméstico. ${ }^{227}$

\footnotetext{
222 Por ejemplo, el artículo 26 de la Constitución de Sudáfrica establece el derecho a una vivienda adecuada, y el artículo 27 establece el derecho a acceder a la atención médica, la alimentación, el agua potable, y la seguridad social. La Constitución establece además que el Estado debe tomar medidas legislativas y de otro tipo que sean razonables, y que estén dentro de los recursos de los que el Estado dispone, para así lograr la progresiva realización de cada uno de estos derechos (artículos 26 (2) y 27 (2), respectivamente).

223 El Poder Judicial sudafricano revisa el cumplimiento de la efectivización progresiva de los artículos 26 y 27 basándose en el test de razonabilidad, tal como se muestra en los casos Government of the Republic of South Africa v Grootboom, 2001 (1) SA 46 (CC), y Minister of Health v. Treatment Action Campaign (no 2) (TAC), 2002 (5) SA 721 (CC).

224 Ortwein, 2003, p. 414.

225 Bull, 2011, pp.393- 420.

226 Op. Cit., Ortwein, p. 414.

227 Comité de Derechos Económicos, Sociales y Culturales, 2016, pár. 5.
} 
No obstante, Suecia ha dado un paso importante recientemente al incorporar parcialmente la Convención de las Naciones Unidas sobre los Derechos del Niño (CDN) al derecho nacional,228 a través de legislación doméstica aprobada en 2018 y vigente desde enero de 2020. Esta nueva ley aclara que las Cortes y los profesionales del derecho deben considerar los derechos contenidos en la Convención sobre los Derechos del Niño y que los derechos de los niños y niñas deben ser considerados en las deliberaciones y evaluaciones realizadas en los procesos de toma de decisiones en los casos y asuntos que conciernan a ellos.229 Si bien todavía no está claro hasta qué grado los derechos de la CDN serán ejecutables por la Corte, existe un proceso tripartito en curso que apoyará la aplicación de la CDN mediante la creación de capacidades, una auditoría jurídica que evalúa las necesidades de cambio para cumplir con la CDN (mapeando las carencias y cómo abordarlas) y la elaboración de una amplia orientación para ayudar a los responsables de la toma de decisiones durante la vigencia de la ley.

Cotidianamente, existen algunos conceptos erróneos sobre la garantía judicial de los DESCA que resultan útiles para reflexionar en un debate informado sobre la incorporación de los DESCA en la nueva Constitución de Chile. Estos conceptos erróneos evidencian preocupaciones legítimas sobre la viabilidad o legitimidad del Poder Judicial o la eventual obstaculización de las decisiones que deberían mantenerse dentro del ámbito del Poder Legislativo o del Poder Ejecutivo. Sin embargo, como lo demuestran los estudios de casos proporcionados en este documento, estas críticas pueden mitigarse y superarse a través de modelos de adjudicación que sean bien concebidos y constitucionalmente apropiados.

En primer lugar, la crítica antidemocrática se refiere a que los tribunales de justicia no pueden interferir legítimamente en la adjudicación de los DESCA pues éstos derechos dependen de los recursos económicos del Estado, cuestión que en una sociedad democrática corresponde decidir única y exclusivamente al Poder Legislativo y al Ejecutivo a través del desarrollo de políticas públicas. Sin embargo, esta crítica omite el hecho de que los derechos civiles y políticos también dependen de los recursos económicos, y a veces, también requieren que la Corte intervenga como mecanismo de rendición de cuentas. Por ejemplo, la instalación de locales de votación para implementar el derecho a voto de las personas privadas de libertad re-
E.

\section{Desafios conceptuales $y$ cómo superarlos}

228 Government Offices of Sweden, 2018.

229 Ibíd. 
quiere de una serie de inversiones de recursos económicos y humanos que no siempre parecen tan evidentes, ${ }^{230}$ mientras que la intervención judicial en materia de vivienda no significa que los jueces desarrollarán las políticas públicas en materia de vivienda, sino que lo que se busca es aprovechar la experiencia de los jueces en los casos concretos, a través de su participación en el debate democrático como fuerza complementaria. Así, el rol coadyuvante de la judicatura en la ejecución de los DESCA es parte importante de un diálogo multiinstitucional que garantiza la rendición de cuentas del poder público en vez de una atribución indebida de poder político a la judicatura.

Por otra parte, la crítica de indeterminación en la adjudicación de los DESCA establece que estos derechos son demasiado imprecisos y vagos, de modo que su interpretación sustantiva no debe dejarse en manos de jueces o Comités de las Naciones Unidas (no electos) sino al amparo de los órganos elegidos democráticamente. Sin embargo, al igual que los derechos civiles y políticos exigen una interpretación, los DESCA también la requieren. En este sentido, los tribunales pueden desempeñar un papel importante en la adjudicación de contenido y propósito a los DESCA, de la misma manera en la que lo hacen con los derechos civiles y políticos. Esto no implica que la judicatura usurpe las funciones de la legislatura o el Poder Ejecutivo; por el contrario, si la legislatura da instrucciones claras a las Cortes sobre cómo interpretar los derechos, éstas pueden ayudar a cumplir la función de garantía de derechos, evitando así la abdicación de esta importante función judicial.

Otra preocupación que a veces surge en quienes rechazan la justiciabilidad de los DESCA, es que en su determinación no existe un órgano judicial o cuasi-judicial a nivel internacional o regional que guíe la interpretación de estos derechos. Sin embargo, esta preocupación no está respaldada por la evidencia. Al contrario, hoy en día existen mecanismos regionales e internacionales para asistir en la interpretación de los DESCA, como por ejemplo, el Protocolo Facultativo del PIDESC, que establece un procedimiento de reclamo en caso de violación de estos derechos a cargo del Comité de Derechos Económicos, Sociales y Culturales. Si bien Chile aún no ha adherido a este mecanismo de denuncias internacionales, los casos resueltos por los órganos de tratados pueden actuar como fuentes útiles de interpretación uniforme. Asimismo, otras cortes regionales, como la Corte Interamericana de Derechos Humanos, poseen un amplio desarrollo en materia de DESCA, pudiendo su jurisprudencia actuar como una herramienta interpretativa. ${ }^{231}$

\footnotetext{
230 Instituto Nacional de Derechos Humanos, 2017.

231 Corte Interamericana de Derechos Humanos, sin fecha.
} 
De igual forma, otras fuentes de interpretación pueden incluir a los Comentarios Generales de los órganos creados en virtud de tratados de las Naciones Unidas, decisiones y recomendaciones de los órganos de tratados y la jurisprudencia de otras jurisdicciones, como las que se han discutido anteriormente.

Por último, la crítica de capacidad señala que los tribunales no tienen la capacidad técnica ni administrativa para manejar los DESCA; que habría una avalancha de litigios, y que los jueces no tienen los conocimientos especializados como para determinar el contenido de estos derechos o su compleja relación con otras esferas del gobierno. Una vez más, de la misma manera que los derechos civiles y políticos están sujetos a adjudicación, existen normas similares que pueden aplicarse en relación con los DESCA. Por ejemplo, el exceso de casos puede evitarse mediante litigios colectivos, enfoques como el de "prueba y suspensión"232 utilizado en el sistema judicial escocés, o los nuevos procedimientos previstos en la Ley de Litigación Civil Escocesa de 2018. ${ }^{233}$ Asimismo, las cortes también pueden contribuir al reforzamiento de su capacidad buscando opiniones expertas sobre los DESCA cuando sea necesario, incluyendo el nombramiento de un amicus curiae (un "amigo de la corte") si se requiere o a la incorporación de clerks (abogados asistentes) dentro del diseño institucional.

Así, la cuestión de si se le debe otorgar a los tribunales un rol en la adjudicación de los DESCA, resulta ser más bien una cuestión de voluntad política más que un problema conceptual. ${ }^{234}$

El proceso constituyente chileno ofrece una oportunidad única para avanzar en la protección de derechos y para actuar como un líder mundial en el desarrollo de vías de protección y el acceso a remedios eficaces cuando ocurran violaciones de derechos sociales. Esta incorporación de los DESCA junto a la adopción de remedios eficaces constituye una manera de abordar la falta de rendición de cuentas en materia de derechos.

Los derechos sociales han estado en el centro de los reclamos por dignidad en Chile, ${ }^{235}$ a la vez que la pandemia del COVID-19 también ofrece un escenario para reflexionar sobre sus secuelas y legado. En tiempos de crisis,

\footnotetext{
232 Scottish Courts, sin fecha, p. 41.

233 Civil Litigation (Expenses and Group Proceedings) (Scotland) Act 2018.

234 Wolffe, 2014, p. 12.

235 IPSOS y Espacio Público, 2021, p. 4.
}

F.

\section{Reflexiones} finales 
los derechos humanos adquieren más relevancia que nunca, sobre todo para garantizar una vida digna a las personas vulneradas por la sociedad en temas tan diversos como la educación, la salud, la vivienda, el agua potable, el saneamiento y la seguridad social. Es en este sentido que la reflexión crítica se hace fundamental no sólo sobre el proceso de cambio constitucional sino también sobre los resultados de aquel proceso.

Es en este sentido que una tarea tan importante como la redacción de la nueva constitución requiere un proceso justo y deliberativo que permita una amplia participación con respecto a su contenido. En este contexto, resulta clave identificar los puntos en que se jugará la garantía real y futura de los DESCA, los que radican en elementos como la transversalización del enfoque de género, la consolidación de los derechos humanos ambientales $y$, por supuesto, los mecanismos que la Convención elija para incorporar domésticamente la aplicación práctica de los derechos humanos.

Si bien el estado crucial en el que se encuentra Chile actualmente tiene muchos puntos de innovación, procesos constituyentes con convenciones constitucionales ya han sido adoptados anteriormente por diversas poblaciones en Latinoamérica, por las provincias canadienses de Columbia Británica y Ontario, por los Países Bajos, Irlanda, Colombia, Islandia, y Escocia. Estos procesos ofrecen ejemplos concretos para el proceso chileno, ${ }^{236} \mathrm{y}$ su recopilación y análisis permiten cimentar un nuevo compromiso constitucional con los DESCA a partir de deliberaciones basadas en evidencia, conforme a la que también debiese guiarse cualquier modelo futuro sobre marcos normativos.

En particular, es importante destacar la necesidad de que la Convención Constitucional chilena desarrolle una discusión sobre la incorporación doméstica de los derechos sociales, y en particular de su justiciabilidad, en atención a que los argumentos históricos que la rechazan han sido refutados por gran parte de la doctrina y la jurisprudencia a nivel mundial. Aquí radica el aporte que este artículo ha pretendido hacer, presentando evidencia comparada y formas de argumentación para contrarrestar formas clásicas de crítica a los DESCA, así como los diversos modelos de incorporación de derechos humanos que otros países han adoptado, con el fin de facilitar la ardua tarea de los y las constituyentes de cara a la elaboración de una Constitución con derechos sociales para el futuro. 

Bull, T. (2011)

'Judges without a court - Judicial Preview in Sweden, Judicial Constitutional Review as a Last Resort', en

Campbell, T. et al. (ed.)

The Legal Protection of Human Rights: Sceptical Essays.

Oxford Scholarship Online.

Carrera, L. (2011)

'La acción de tutela en Colombia', Revista del Instituto de Ciencias Jurídicas de Puebla, V(27).

Civil Litigation (Expenses and Group Proceedings) (Scotland)

Act 2018 (asp 10). Disponible en: https://www.legislation.gov.uk/ asp/2018/10/section/20/enacted (Consulta: 13 diciembre 2020).

Comité de Derechos del Niño (2003)

Observación general nro. 5 (2003): Medidas generales de aplicación de la Convención sobre los Derechos del Niño. Documento No. CRC/GC/2003/5, 27 noviembre. Disponible en: https://undocs.org/es/CRC/GC/2003/5 (Consulta: 14 diciembre 2020).

Comité de Derechos Económicos, Sociales y Culturales (1998) Proyecto de Observación general N 9. La aplicación interna del Pacto. A. El deber de dar efecto al Pacto en el ordenamiento jurídico interno.

Comité de Derechos Económicos, Sociales y Culturales (2014) Observaciones finales sobre el sexto informe periódico

Comité de Derechos Económicos, Sociales y Culturales (2015) Observaciones finales sobre el cuarto informe periódico de Chile. Resolución no. E/C.12/CHL/CO/4, 7 julio. Disponible en: http://docstore.ohchr.org/SelfServices/FilesHandler.ashx? enc=4slQ6QSmlBEDzFEovLCuW\%2BALqOml1btoJd4Yx REVF2VLpsynzEQ14MrIM81mGrQY8i6Ffm4Xu53oEyZ9iXXvgu \%2FuulQCVPaTThiVh8Lj2INqlOCWrfwuL3PuElm\%2BZhxX (Consulta: 13 diciembre 2020).

Comité de Derechos Económicos, Sociales y Culturales (2016) Observaciones finales relativas al sexto informe periódico de Suecia. Documento No. E/C.12/SWE/CO/6, 14 julio. Disponible en: https://tbinternet.ohchr.org/_layouts/15/treatybodyexternal/ Download.aspx?symbolno=E/C.12/SWE/CO/6\&Lang=En (Consulta: 14 diciembre 2020). 
Conferencia Mundial de Derechos Humanos (1993)

Declaración y Programa de Acción de Viena, p. 19. Disponible en: https://www.ohchr.org/documents/events/ohchr20/ vdpa_booklet_spanish.pdf (Consulta: 11 noviembre 2020).

Corte Interamericana de Derechos Humanos (sin fecha) Sentencias. Disponible en: https://www.corteidh.or.cr/casos_sentencias.cfm (Consulta: 13 diciembre 2020).

Craven, M. (1995)

The International Covenant on Economic, Social, and Cultural Rights, A Perspective on its Development. Clarendon Press.

De Búrca, G. (2012)

'The Future of Social Rights Protection in Europe', en De Búrca, G. et al. (ed.) Social Rights in Europe. Oxford Scholarship Online.

De Finlandia. Resolución no. E/C.12/FIN/CO/6, 17 diciembre. Disponible en: https://undocs.org/es/E/C.12/FIN/CO/6 (Consulta: 14 diciembre 2020).

Farrell, D. (2014)

'The 2013 Irish Constitutional Convention: A Bold Step or a Damp Squib?', in Ferrari, G. y O'Dowd, J. (ed.) 75 Years of the Constitution of Ireland: An Irish-Italian Dialogue. Clarus Press.

Disponible en: http://www.openpolitics.ro/wp-content/uploads/ UCD_Mar13_final1.pdf

(Consulta: 13 diciembre 2020).

Figueroa, P. Y Jordán, T. (2020)

7 propuestas para la nueva constitución de Chile. Santiago: Universidad de Santiago de Chile.

First Minister's Advisory Group on Human Rights Leadership (sin fecha) About us. Disponible en: https://humanrightsleadership.scot/ (Consulta: 14 diciembre 2020).

Gauri, V. y Brinks, D. (2010)

Courting Social Justice: Judicial Enforcement of Social and Economic Rights in the Developing World.

Cambridge University Press.

Gearty, C. y Mantouvalou, V. (2011)

Debating Social Rights. Oxford; Portland, Oregon: Hart Publishing. 
Government Offices of Sweden (2018)

Convention on the Rights of the Child will become Swedish law. Disponible en: https://www.government.se/articles/2018/03/ new-legislative-proposal-on-the-convention-on-the-rights-ofthe-child/

(Consulta: 13 diciembre 2020).

Instituto Nacional de Derechos Humanos (2017)

Corte Suprema resuelve que personas privadas de libertad pueden votar. Disponible en: https://www.indh.cl/cortesuprema-resuelve-que-personas-privadas-de-libertadpueden-votar/ (Consulta: 13 diciembre 2020).

IPSOS y Espacio Público (2021)

¿Cómo vemos el proceso constituyente? Miradas a un momento histórico. Informe No 1 - Indagación cualitativa. Disponible en: https://www.ipsos.com/sites/default/files/ct/news/ documents/2021-03/ppt-bhp-informe-n1-final.pdf (Consulta: 21 junio 2021).

Koch, I. (2005)

'Dichotomies, Trichotomies or Waves of Duties', Human Rights Law Review, 5(1). DOI: 10.1093/hrlrev/ngi004.

Minkler, L. (2013)

The State of Economic and Social Human Rights:

A Global Overview. Cambridge University Press.

Ortwein, B (2003)

'The Swedish Legal System: An Introduction', Indiana International \& Comparative Law Review, 13(2).

Disponible en: https://journals.iupui.edu/index.php/iiclr/article/ view/17766/17949

(Consulta: 13 diciembre 2020).

Resnik, J. (2006)

'Law's Migration: American Exceptionalism, Silent Dialogues, and Federalism's Multiple Ports of Entry', The Yale Law Journal, 115. Disponible en: https://digitalcommons.law.yale.edu/cgi/ viewcontent.cgi?article $=5017 \&$ context $=y l j$

(Consulta: 14 diciembre 2020).

Scottish Courts (sin fecha)

The Symmary Cause. Information and procedural guide.

Disponible en: https://www.scotcourts.gov.uk/docs/

default-source/rules-and-practice/guidance-notes/gn---sum

mary-cause/4-going-to-court---cases-raised-on-or-after-1-

december-2009.doc?sfvrsn=34

(Consulta: 13 diciembre 2020). 
Scottish Government (sin fecha)

Programme for Governement. Disponible en: https://beta.gov. scot/programme-for-government/

(Consulta: 14 diciembre 2020).

Scottish Human Rights Commission (2015)

Creating a Fairer Scotland: A Human Rights Based Approach to Tackling Poverty.

Disponible en: https://www.scottishhumanrights.com/ media/1266/fairerscotlandsep2015.pdf

(Consulta: 13 diciembre 2020).

Sepúlveda, M. (2009)

'Colombia: The Constitutional Court's Role in Addressing Social Injustice", en Langford, M. (ed.) Social Rights Jurisprudence, Emerging Trends in International and Comparative Law.

Skelton, A. (2010)

'Girls' Socio-Economic Rights in South Africa', South African Journal of Human Rights, 26(1).

Tierney, S. (2012)

Constitutional Referendums: The Theory and Practice of Republican Deliberation. Oxford University Press.

Tierney, S. y Boyle, K. (2013)

'Yes or No, Scotland's Referendum Carries Significant Constitutional Implications', Scottish Constitutional Futures Forum, 14 noviembre.

Disponible en: https://www.scottishconstitutionalfutures.org/ OpinionandAnalysis/ViewBlogPost/tabid/1767/articleType/ ArticleView/articleld/2550/Stephen-Tierney-and-KatieBoyle-Yes-or-No-Scotlands-Referendum-Carries-SignificantConstitutional-Implications.aspx (Consulta: 14 diciembre 2020).

Wolffe, J. (2014)

'Economic and Social Rights in Scotland: Lessons from the Past; Options for the Future'. A lecture for International Human Rights Day 2014. Universidad de Edimburgo.

Disponible en: https://www.scottishhumanrights.com/ media/1469/wolffe2014lecture.pdf

(Consulta: 13 diciembre 2020). 